\title{
Avaliação físico-química e atividades biológicas do extrato de uvaia (Eugenia pyriformis Camb.)
}

\author{
Antonio Carlos Pereira de Menezes Filho ${ }^{\mathrm{a} *(\mathbb{0}}$ \\ a Instituto Federal de Educação, Ciência e Tecnologia Goiano, Rio Verde, Goiás, Brasil \\ *Autor correspondente (astronomoamadorgoias@gmail.com)
}

\section{N F O}

Keyworks

Myrtaceae

ripe fruits

photoprotective activity

hemolytic activity
Palavras-chaves

Myrtaceae

fruto maduro

atividade fotoprotetora

atividade hemolítica

\begin{abstract}
A B S T R A C T
Physicochemical evaluation and biological activities of the extract uvaia (Eugenia pyriformis Camb.) The Myrtaceae family has numerous fruit genera such as Eugenia. The aim of this work was to evaluate the hydroethanolic extract of the ripe fruit of Eugenia pyriformis on the physicochemical characteristics and photoprotection and cytotoxic on human erythrocytes. The ripe fruits were collected and pulped. The extract was produced by maceration in a $70 \%$ hydroethanolic solution. The physical-chemical characterization was performed for qualitative sensory analysis, extract yield, dry mass, water content, $\mathrm{pH}$, total solids, refractive index, relative density, total flavonoid and tannin contents, thin layer chromatography, scanning between 400 at $900 \mathrm{~nm}$, photoprotection scanning activity between 220 to $400 \mathrm{~nm}$ and hemolytic activity on human erythrocytes and different concentrations of extract. The results showed yellow, homogeneous and aromatic color for the extract. Yield of $20.05 \%$, dry mass $16.18 \%$, water content $83.82 \%, \mathrm{pH} 2.64$, total solids $2.07 \%$, refractive index $1.4025 \mathrm{n}_{\mathrm{D}}$, density $1.013 \mathrm{~g}$ $\mathrm{mL}^{-1}$, flavonoids $72.87 \mathrm{mg} \mathrm{RU} 100 \mathrm{~g}^{-1}$, tannins $6.05 \mathrm{mg} \mathrm{CA} 100 \mathrm{~g}^{-1}$, several $\mathrm{R} f \mathrm{~s}$, with the acetone/chloroform elotropic set having a greater number of isolated compounds, presence of natural sugars glucose and sucrose. UVC and cytotoxic photoprotection, activity on erythrocytes between $35.04 \%$ to $2.14 \%$. The results showed safety on the use of the hydroethanolic extract of the fruit of $E$. pyriformis being able to be used as a photoprotective solution of the natural line. Further studies should be carried out evaluating other biological activities by extracting the fruit of Eugenia pyriformis.
\end{abstract}




\section{INTRODUÇÃO}

O Brasil, segundo Ferreira et al. (2011) e Dexheimer e Pozzobon (2017), apresenta uma alta porcentagem de espécies pertencentes à flora mundial, sendo inúmeras espécies endêmicas dos mais variados biomas e domínio Cerrado brasileiro. No entanto, sabe-se que menos de $10 \%$ desse contingente florístico foi estudado, e menos de 5\% apresentam algum estudo fitoquímico, bem como, ações ou atividades biológicas inexistentes em estudos científicos.

Os vegetais medicinais ora raízes, caules, cascas, talos, folhas, flores, frutos e sementes apresentam uma rica constituição fitoquímica em cada órgão vegetal, sendo produzidos naturalmente por processos fisiológicos contínuos. A essa constituição, dá-se o nome de compostos metabólicos secundários, sendo várias fitomoléculas desses metabólitos usadas no tratamento e cura de inúmeros problemas infecciosos que afligem a humanidade até os dias atuais (Zapata et al., 2018).

A família Myrtaceae é uma de várias grandes famílias botânicas encontradas nas Américas, principalmente na América do Sul. São conhecidos aproximadamente 144 gêneros e mais de 4.630 espécies, com dois centros de distribuição na América tropical e Austrália, e nas regiões tropicais e subtropicais do mundo (Arantes e Monteiro, 2002, Silva e Mazine, 2016). No Brasil, são descritas 1.034 espécies dentro de 23 gêneros (Silva; Mazine, 2016; Lazzarotto-Figueiró et al., 2021). Esta família está inserida na ordem Myrtales abrangendo entorno de $40 \%$ do total das espécies da ordem. Constantemente, a família Myrtaceae passa por reclassificações intrafamiliares, através de ensaios moleculares e morfológicos buscando conhecer melhor as características macro e microestruturais nas relações filogenéticas (Lucas et al., 2007, 2011; Silva e Mazine, 2016).

O gênero Eugenia é bem representado dentro de Myrtaceae, com aproximadamente 1.000 espécies descritas. A espécie Eugenia pyriformis Cambess., Fl. Bras. Merid. (A. St. -Hil.). ii. 336. 1933 é conhecida popularmente por "uvalha, uvalha do campo e uvaia" apresentando porte arbóreo de até $5 \mathrm{~m}$ de altura, apresentando indumento castanhoamarelado ou bronzeado; os ramos adultos apresentam coloração castanho-acinzentados ou castanho-avermelhados; as folhas apresentam pecíolo entre $2-4 \mathrm{~mm}$ de compr., de coloração verde-clara; lâminas entre 12-40 x 5-12 mm, lanceoladas ou estreito-elípticas; flores em dicásio, axilares; frutos do tipo baga piriformes, não costadas, amarelas quando maduras, apresentando
1-2 sementes. A espécie ocorre desde o Brasil até o Paraguai, Uruguai e Norte da Argentina, sendo esta uma espécie nativa do bioma de Mata Atlântica e do domínio Cerrado (Silva e Mazine, 2016).

Ainda pouco se conhece sobre E. pyriformis, sendo que, os trabalhos são escassos e a linha de publicação muito tênue. Os poucos estudos apresentam para a caracterização do fruto quanto a sua constituição físico-química, excepcional conteúdo de vitamina $\mathrm{C}$, de compostos fenólicos totais, com alta atividade antioxidante, importantes teores de carotenoides e minerais, sendo estes, expressivos, dentre outros. Embora os frutos de uvaia forem ainda pouco explorados quanto a sua possível empregabilidade e diversidade de formas na alimentação, e ainda para diversas atividades biológicas tanto in vitro e/ou in vivo, estudos devem ser realizados avaliando o fruto in natura, bem como seus extratos e frações, determinando assim o seu potencial nutritivo e/ou farmacológico (Kauffmann et al., 2017; Machado et al., 2019; Bianchini et al., 2020).

Quanto às atividades de fotoproteção e citotoxicidade sobre eritrócitos humanos, vários extratos vegetais apresentam ação em diferentes comprimentos de ondas absorvendo energias no ultravioleta (UV) (A, B ou C) servindo como filtros solares naturais com ação de proteção, absorção, e/ou refletindo ou refratando a radiação ultravioleta. Assim, o fotoprotetor tem sua ação biológica na proteção contra agravos na pele podendo ser um simples eritema a prevenção de um carcinoma (Violante et al., 2009; Catelan et al., 2019). Quanto aos efeitos citotóxicos sobre eritrócitos humanos, alguns extratos vegetais podem em ensaios in vitro, ser observado reação citotóxica em diferentes graus (alta, leve, moderada e baixa), que podem restringir o uso destes como meio fitoterapêutico (Paunović et al., 2016; Ramos et al., 2020).

O trabalho teve por objetivo, avaliar o extrato hidroetanólico $70 \%$ do fruto de Eugenia pyriformis quanto às características físicoquímicas e atividades biológicas sobre ação fotoprotetora e citotoxicidade em eritrócitos.

\section{MATERIAL E MÉTODOS}

\section{Coleta dos frutos}

Os frutos de E. pyriformis foram coletados no mês de outubro de 2020 em uma propriedade rural localizada no município de Rio Verde, estado de Goiás, Brasil com a seguinte coordenada 


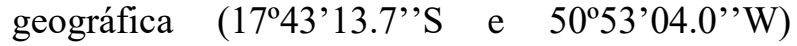
(Garmin, Mod. GPSMAP 64x GPS/Glonass). Um total de $1 \mathrm{~kg}$ de frutos, foram colhidos $\mathrm{e}$ armazenados em embalagem plástica mantida sob resfriamento, e em seguida, transportada para o laboratório de Química Tecnológica no Instituto Federal Goiano, Campus Rio Verde.

A espécie foi identificada pelo Biólogo $\mathrm{Msc}^{\circ}$. Antonio Carlos Pereira de Menezes Filho, e em seguida, uma exsicata foi herborizada e depositada no Herbário do laboratório de Sistemática Vegetal do IF Goiano, Campus Rio Verde, com o Voucher HRV 12097.

\section{Produção do extrato}

Os frutos foram inicialmente limpos em uma solução aquosa de hipoclorito de sódio a $1 \%(v / v)$ por 15 minutos, e logo em seguida, lavados em água corrente e deixados sob papel toalha para retirada do excesso de água.

O extrato hidroetanólico $70 \%$ foi preparado com $200 \mathrm{~g}$ de fruto (casca com polpa), e as sementes descartadas. $\mathrm{O}$ processo utilizado foi $\mathrm{o}$ de maceração por 10 dias em local escuro com temperatura ambiente. Após esse período, o extrato foi filtrado em papel de filtro qualitativo faixa azul (Unifil), e o sobrenadante recolhido e reduzido em rotaevaporador rotativo com pressão negativa (Fisatom, Mod. 803). Após esse processo, o extrato foi liofilizado em liofilizador (Liotop, Mod. L 101) conforme descrito por Amorim et al. (2020).

\section{Ensaios físico-químicos}

A análise sensorial organoléptica foi realizada para cor, aspecto homogêneo e/ou heterogêneo e aroma ou ausência deste. A massa do extrato foi determinada utilizando balança analítica digital (Shimadzu, ATY224), e o rendimento determinado em percentagem conforme equação 1, descrita por Alves et al. (2011).

$\operatorname{Red} \%=[(\mathrm{g}$ extrato seco $\times 100) / \mathrm{g}$ droga vegetal $]$ Eq. [1]

A massa seca e teor de umidade foram determinados utilizando $100 \mathrm{~g}$ de casca com polpa do fruto de uvaia. Para umidade foi utilizado método gravimétrico conforme descrito por Franzen et al. (2018), através da diferença de massa em estufa com circulação de ar forçada (Thoth, Mod. 510) a $105^{\circ} \mathrm{C}$ até massa constante, e a massa seca total determinada pela diferença do resultado de umidade da amostra in natura.

$\mathrm{O} \mathrm{pH}$ foi determinado em pHmetro digital
(Lucadena, Mod. 2010-P). Onde, uma alíquota de $50 \mathrm{~mL}$ de extrato foi utilizada para a análise. O conteúdo de sólidos totais foi determinado em estufa com circulação de ar forçada (Nova Ética, Mod. $400-3 \mathrm{ND}$ ) a $105{ }^{\circ} \mathrm{C}$ por 3 horas. O resíduo após aquecimento foi resfriado e em seguida, a massa foi determinada em balança analítica digital. O resultado foi expresso em percentagem, conforme proposto por Cala-Calviño et al. (2018).

$\mathrm{O}$ índice de refração foi obtido em refratômetro digital (Hanna Instruments, Mod. HI96800), com faixa entre 1,3330 a $1,5080 \mathrm{n}_{\mathrm{D}}$. Foi utilizado 3 gotas do extrato hidroetanólico para determinação (CALA-CALVIÑO et al., 2018). A densidade relativa foi determinada em proveta graduada (Vidrolabor) de $10 \mathrm{~mL}$, e o resultado expresso em g $\mathrm{mL}^{-1} 25{ }^{\circ} \mathrm{C}$. Inicialmente, a proveta foi previamente seca em dessecadora com sílica gel por $24 \mathrm{~h}$, e em seguida pesada. Logo após, foi acrescida com $10 \mathrm{~mL}$ de extrato, e posteriormente pesada em balança analítica digital, conforme descrito por Domínguez et al. (2019), adaptado.

$\mathrm{O}$ teor de flavonoides totais foi determinado pelo método descrito por Zhishen et al. (1999) e Sganzerla et al. (2019), e proposto por Bianchini et al. (2020) adaptado. Foram adicionados $500 \mu \mathrm{L}$ de extrato em $0,15 \mathrm{~mL}$ de uma solução aquosa de nitrito de sódio conc. de $5 \%(\mathrm{~m} / \mathrm{v})$. Em seguida, a solução foi homogeneizada em Vortex e mantida em repouso em temperatura ambiente por 5 minutos em local escuro. Logo após, foi adicionado $0,15 \mathrm{~mL}$ de uma solução aquosa de cloreto de alumínio com conc. $10 \%(\mathrm{~m} / \mathrm{v})$, seguida de incubação por 6 minutos. Após esse período, foi adicionado $1000 \mu \mathrm{L}$ de uma solução aquosa de hidróxido de sódio com conc. de $1 \mathrm{Mol} \mathrm{L}^{-1}$. A leitura foi realizada em espectrofotômetro UV-Vis (Belphotonics, Mod. M-51) no comprimento de ondas em $425 \mathrm{~nm}$ utilizando cubeta de quartzo de campo único de $1 \mathrm{~cm}$. O resultado foi expresso em $\mathrm{mg}$ de rutina (Purifarma, P.A -ACS) (RU) $100 \mathrm{~g}^{-1}$ de amostra. Uma curva padrão de quercetina foi realizada para avaliação dos resultados.

O conteúdo de taninos totais foi determinado utilizando o método descrito por Morrison et al. (1995) adaptado conforme descrito por Vieira e Orlanda (2018). Em tubo de ensaio foi depositado uma alíquota de $0,1 \mathrm{~mL}$ do extrato de uvaia, em seguida adicionou-se $0,9 \mathrm{~mL}$ de álcool metílico (P.A - ACS), 2,5 mL de uma solução metanólica de vanilina conc. $2,25 \mathrm{~mol} \mathrm{~L}^{-1}$. A solução foi aquecida em banho-maria a $60{ }^{\circ} \mathrm{C}$ por $10 \mathrm{~min}$. Logo após, foi realizada leitura em espectrofotômetro UV-Vis em $500 \mathrm{~nm}$ utilizando cubeta de vidro óptico. Uma curva de calibração foi realizada utilizando catequina (P.A - ACS) entre 0,01 a $700 \mu \mathrm{g} \mathrm{mL}^{-1}$. Os resultados foram 
expressos em mg equivalentes em catequina (CA) $100 \mathrm{~g}^{-1}$ do extrato liofilizado.

Uma varredura foi realizada no extrato entre os comprimentos de ondas 900 a $400 \mathrm{~nm}$ em espectrofotômetro UV-Vis (Belphotonics, Mod. M-51), utilizando cubeta de quartzo com campo óptico de $1,0 \mathrm{~cm}$. A caracterização cromatográfica foi realizada em camada delgada (CCD). Para isso, foi utilizada placa cromatográfica (DCFertigfolien Alugran ${ }^{\circledR}$ Xtra SIL G/UV 254$)$, cortada em tiras com as seguintes dimensões $(1 \times 10 \mathrm{~cm})$.

As fases móveis utilizadas foram: acetona/clorofórmio e acetato de etila/acetona. E para a revelação das classes fitoquímicas, foram utilizadas soluções de vanilina sulfúrica, luz ultravioleta nos comprimentos de ondas curto e longo $\left(U_{254}\right.$ e $\left.U V_{365} \mathrm{~nm}\right)$, solução aquosa de cloreto férrico, solução de ácido crômico, vapor de Iodo sublimado, solução aquosa de permanganato de potássio estabilizado, solução de verde de bromocresol e solução de difenilamina ácida.

Os açúcares redutores e não redutores, foram identificados qualitativamente utilizando padrões (P.A - ACS) de glicose, frutose, xilose, dextrina e sacarose a $1 \%$ em solução aquosa, e revelados com solução cetônica de difenilamina sulfúrica $1 \%(\mathrm{~m} / \mathrm{v})$ conforme descrito por Silva et al. (2003) utilizando fitas de cromatoplacas como descrito anteriormente. $\mathrm{O}$ conjunto de eluidores utilizado foi acetona e clorofórmio (1:1). $O$ fator de retenção expresso em (Rfs) foi determinado utilizando régua milimétrica, conforme descrito por Alves et al. (2011).

\section{Atividades biológicas}

A determinação do comprimento de ondas crítico (fotoprotetor) foi realizada com uma varredura em espectrofotômetro UV-Vis (Belphotonics, Mod. M-51) nas regiões ultravioleta A, B e C (UVA, UVB e UVC) entre 100 a $400 \mathrm{~nm}$ conforme descrito por Violante et al. (2009) e Menezes Filho et al. (2020) adaptado, utilizando o extrato hidroetanólico.

A atividade hemolítica sobre eritrócitos humanos foi realizada conforme descrito por Ramos et al. (2020). Onde em três tubos Falcon cônicos (Sarstedt) de $50 \mathrm{~mL}$, foram acrescidos 5 $\mathrm{mL}$ de solução fisiológica $\mathrm{NaCl}$ concentração 0,9\% (Arboreto/comercial), e três tubos Falcon cônicos como controle positivo contendo água destilada e tecido hematopoiético a $5 \%$ em solução salina $0,85 \%$.

O ensaio foi realizado nas concentrações entre 5 a $1000 \mu \mathrm{g} \mathrm{mL}^{-1}$ de extrato hidroetanólico. As amostras foram incubadas em temperatura de 25 ${ }^{\circ} \mathrm{C}$ em banho ultratermostatizado (Solab, Mod. SL 152-I) por $15 \mathrm{~min}$. Logo em seguida, as amostras foram centrifugadas (Fanem, Mod. Excelsa II) a $1000 \mathrm{rpm}$ durante 20 minutos. Em seguida, o sobrenadante foi coletado através de uma pipeta automática de $1000 \mu \mathrm{L}$ e transferido para uma cubeta de vidro óptico de $5 \mathrm{~mL}$, e analizado em espectrofotômetro UV-Vis (Belphotonics, Mod. M-51) no comprimento de ondas fixo de $540 \mathrm{~nm}$ em absorbância.

O percentual hemolítico foi determinado através do (Abs controle positivo) como $100 \%$. O porcentual hemolítico sobre eritrócitos humanos foi determinado conforme equação 3 , proposta por Dacie et al. (1975).

$\% \mathrm{Hem}=\left(\mathrm{Abs}_{\text {amostra }}\right) * 100 /\left(\mathrm{Abs}_{\text {controle }}\right) \quad$ Eq. [3]

Em seguida, todo material utilizado neste ensaio de hemólise, foi inicialmente lavado em uma solução aquosa de hipoclorito de sódio $1 \%$ (v/v) e logo após, foi devidamente autoclavado a $121{ }^{\circ} \mathrm{C} 1 \mathrm{Atm}$, por 25 minutos. Após autoclavagem, o material foi lavado em água corrente e seco em estufa a $105{ }^{\circ} \mathrm{C}$ por 30 minutos.

\section{Análise estatística e tratamento das imagens}

Os experimentos foram realizados em triplicata seguida de \pm desvio padrão, exceto para o ensaio de $C C D$ que foi realizado uma única vez. O programa estatístico utilizado foi o PAST 3 (versão 2019, livre). Para o tratamento das imagens de varredura em espectrofotometria UVVis, foi utilizado o programa Origin (versão 2018, paga).

\section{RESULTADOS E DISCUSSÃO}

A análise sensorial demonstrou um extrato homogêneo, com coloração amarelado ouro, muito aromático característico do fruto de uvaia. $\mathrm{Na}$ Figura 1, está apresentado o fruto de uvaia e o extrato hidroetanólico, respectivamente.

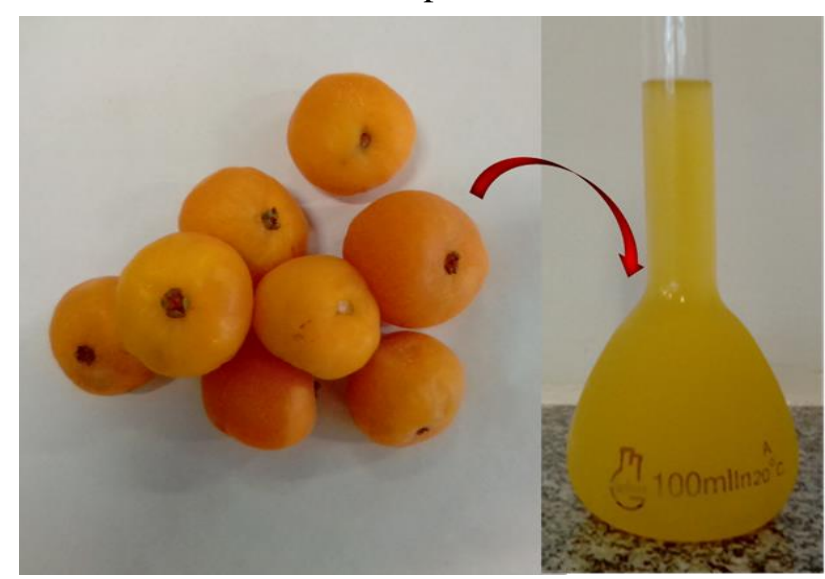

Figura 1 - Frutos maduros e extrato hidroetanólico bruto de Eugenia pyriformis. 
Na tabela 1, estão apresentados os parâmetros físico-químicos obtidos a partir do extrato hidroetanólico da casca com polpa do fruto de uvaia.

Tabela 1 - Parâmetros físico-químicos do extrato hidroetanólico $70 \%$ da casca com polpa de Eugenia pyriformis.

\begin{tabular}{lc}
\hline Parâmetros & ECP* \\
\hline Rendimento extrato (\%) & $20,05 \pm 0,21$ \\
Massa seca (\%) & $16,18 \pm 0,07$ \\
Teor de água (Tu\%) & $83,82 \pm 0,11$ \\
pH & $2,64 \pm 0,11$ \\
Sólidos totais (ST\%) & $2,07 \pm 0,21$ \\
Índice de refração $\left(\mathrm{n}_{\mathrm{D}}\right)$ & $1,4025 \pm 0,05$ \\
Densidade relativa $\left(\mathrm{g} \mathrm{mL}^{-1}\right)$ & $1,013 \pm 0,00$ \\
Flavonoides (mg RU 100 g & $72,87 \pm 0,11$ \\
Taninos (mg CA 100 g $\left.)^{-1}\right)^{* *}$ & $6,05 \pm 0,08$ \\
\hline *ECP = Extrato casca com polpa. ** Análise fitoquímica qualitativa também foi realizada para determinação de taninos (verde) que \\
corresponde a condensados ou catéquicos. Médias seguidas de \pm desvio padrão. Análises realizadas em triplicata seguida de \pm desvio \\
padrão. Tu\% (teor de umidade), ST\% (sólidos totais), RU (equivalentes em rutina) e CA (equivalente em catequina).
\end{tabular}

O rendimento de extrato foi de $20 \%$, massa seca de $16 \%$, teor de água de $83 \%$, pH de 2,64, sólidos totais de $2 \%$, índice de refração de $1,40 \mathrm{n}_{\mathrm{D}}$, densidade de $1,0 \mathrm{~g} \mathrm{~mL}^{-1}$. O teor de flavonoides totais foi expressivo, apresentando resultado igual a 72 $\mathrm{mg}$ equivalentes em rutina $100 \mathrm{~g}^{-1}$ de extrato hidroetanólico de uvaia liofilizado. Já para o conteúdo de taninos totais (condensados ou catéquicos) apresentou baixo teor igual a $6 \mathrm{mg}$ equivalentes em catequina $100 \mathrm{~g}^{-1}$ de extrato hidroetanólico de uvaia liofilizado. A presença de flavonoides e taninos no extrato de uvaia sugere elevado potencial antioxidante, principalmente por atuarem co- mo sequestradores de radicais livres como o oxigênio singleto e como fotoprotetores contra os raios UV (Vieira e Orlanda, 2018). Resultados similares foram observados por Plucenio e Silva (2017) onde avaliaram o fruto e o extrato onde relatam para flavonoides teor de 6,5 e 4,67 mg EQ $100 \mathrm{~g}^{-1}$, respectivamente.

Na tabela 2, estão apresentados os resultados da caracterização qualitativa por cromatografia em camada delgada (CCD) do extrato hidroetanólico de uvaia em diferentes combinações de eluidores e reveladores.

Tabela 2 - Caracterização do extrato hidroetanólico $70 \%$ da casca com polpa de Eugenia pyriformis a partir do ensaio de cromatografia em camada delgada (CCD).

\begin{tabular}{|c|c|c|c|c|c|c|c|c|}
\hline Eluentes & $\begin{array}{l}\text { UV } \\
254\end{array}$ & $\begin{array}{l}\mathrm{UV} \\
365\end{array}$ & $\begin{array}{l}\text { Vanilina } \\
\text { sulfúrica }\end{array}$ & $\mathrm{FeCl}_{3}$ & $\mathbf{I}_{2}$ & $\mathrm{KMnO}_{4}$ & $\mathrm{C}_{21} \mathrm{H}_{14} \mathrm{Br}_{4} \mathrm{O}_{5} \mathrm{~S}$ & $\mathrm{H}_{2} \mathrm{CrO}_{4}$ \\
\hline 1 & $\begin{array}{l}0,77 \\
0,20\end{array}$ & 0,57 & $\begin{array}{c}0,75^{*} \\
0,73^{*} \\
0,20^{*} \\
0,15^{*}\end{array}$ & 0,20 & $\begin{array}{l}0,80 \\
0,20 \\
0,15\end{array}$ & $\begin{array}{l}0,20 \\
0,17\end{array}$ & $\begin{array}{l}0,85 \\
0,70 \\
0,30 \\
0,15\end{array}$ & $0,15^{*}$ \\
\hline 2 & $\begin{array}{l}0,70 \\
0,20\end{array}$ & 0,55 & $\begin{array}{l}0,75^{*} \\
0,25^{*} \\
0,17^{*}\end{array}$ & 0,20 & $\begin{array}{l}0,80 \\
0,15\end{array}$ & - & $\begin{array}{l}0,82 \\
0,36 \\
0,19\end{array}$ & - \\
\hline
\end{tabular}

$1=$ acetona/clorofórmio. 2 = acetona/acetato de etila. (-) não determinado. *Aquecimento a $95{ }^{\circ} \mathrm{C}$.

Observa-se na tabela 2, que a combinação acetona/clorofórmio apresentou melhor resultado quando comparado à combinação acetona/acetato de etila, onde foram obtidos 18 e $12 \mathrm{R} f s$ separados pela diferença elotrópica, determinando assim, que a acetona e o clorofórmio combinados formam um excelente eluidor para o extrato do fruto de uvaia. Além disso, a vanilina sulfúrica e o verde de bro- mocresol apresentaram o maior número de $\mathrm{R} f s$ identificados em ambos os conjuntos da fase móvel.

Nas cromatoplacas ficou bem evidenciada a presença de flavonoides com cor (marrom) e R $f s$ de $(0,15$ e $0,20 \mathrm{~mm} / 0,17$ e $0,25 \mathrm{~mm})$ utilizando revelador vanilina sulfúrica, e para taninos (verde) $\mathrm{R} f s(0,20 \mathrm{~mm} / 0,20 \mathrm{~mm})$ utilizando o revelador 
cloreto de férrico, respectivamente para acetona/clorofórmio e acetona/acetato de etila. Comparando com outros trabalhos que avaliaram essa técnica e tipo de revelador, Silva et al. (2019), avaliaram o extrato do fruto de jenipapo (Genipa americana), onde encontraram para flavonoides e revelador vanilina sulfúrica também coloração marrom na cromatoplacas com $\mathrm{R} f s$ de $0,10 \mathrm{~mm}$.

Corroborando com o discutido por Wagner e Blandt onde diz que a coloração marrom pode indicar a presença de flavonoides, o que pode ser constatado neste estudo, tanto pela técnica qualitativa quanto quantitativa. Observa-se que vários $\mathrm{R} f s$ estão envolvidos em compostos fenólicos na amostra avaliada. Plucenio e Silva (2017) encontraram expressivo conteúdo de fenólico totais de 34,72 e 49,70 mg EAG $100 \mathrm{~g}^{-1}$ e atividade de redução do radical DPPH com $\mathrm{CE}_{50}$ de 930,78 e $799,85 \mu \mathrm{g}$ EQ $\mathrm{mL}^{-1}$, para fruto e extrato de $E$. pyriformis, respectivamente. No entanto, no estu- do de Stiven, Moreira e Silva (2009) onde avaliaram os extratos etanólico e aquoso do fruto de $E$. pyriformis, os pesquisadores não observaram a presença de compostos fenólicos por teste qualitativo, o mesmo foi observado avaliando a atividade antioxidante pelo método do radical DPPH. Sugere-se que, outros métodos de redução de radicais livres devem ser utilizados comparando assim com o ensaio por DPPH.

A pesquisa de açúcares em CCD demonstrou a presença positiva para glicose e sacarose com $R f s$ de 0,45 e 0,39 $\mathrm{mm}$, e para os padrões de glicose e sacarose anidra com $R f s$ de 0,40 e $0,35 \mathrm{~mm}$, respectivamente.

As figuras 2 e 3 devem ser interpretadas juntas para esse extrato hidroetanólico do fruto de $E$. pyriformis. Na Figura 2, está apresentada uma varredura em espectrofotometria entre 900 a 400 nm.

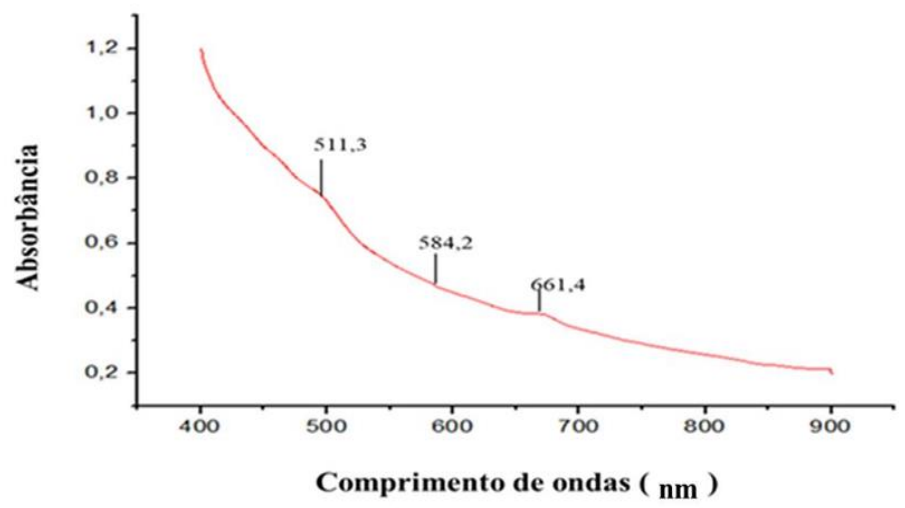

Figura 2 - Varredura por espectrofotometria UV-Vis entre 900 a 400 nm no extrato hidroetanólico de Eugenia pyriformis.

Na Figura 3, está apresentada uma varredura em espectrofotometria entre 400 e $220 \mathrm{~nm}$ consi-

derado como comprimento de ondas crítico.

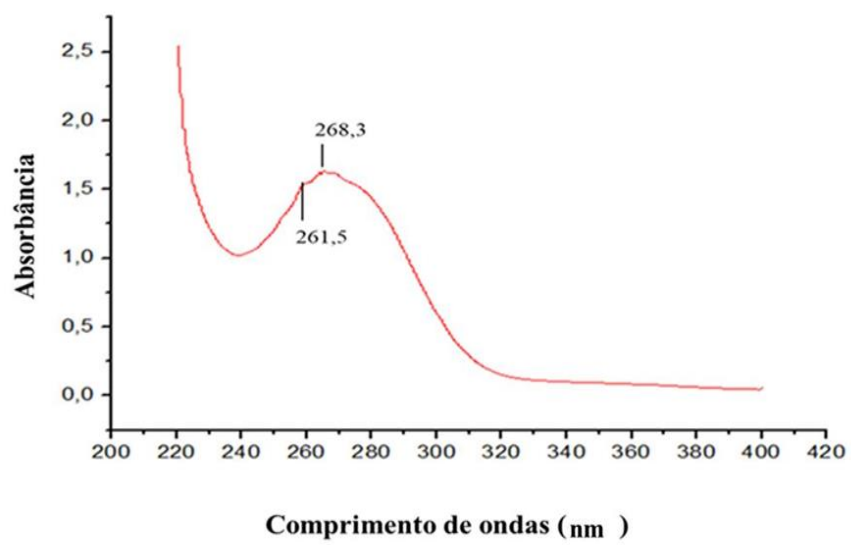

Figura 3 - Comprimento de ondas crítico por varredura em espectrofotometria UV-Vis entre 400 a $220 \mathrm{~nm}$ no extrato hidroetanólico $70 \%$ de Eugenia pyriformis. 
Foi observada uma banda larga e intensa em $268,3 \mathrm{~nm}$ e um braço dessa banda estreito e fraco em 261,5 (Fig. 3) e discretas bandas em 511,3 e $584,2 \mathrm{~nm}$ (Fig. 2) no extrato hidroetanólico de uvaia. Essas bandas podem estar envolvidas na ação de absorção de flavonoides e $\beta$-caroteno (todo-trans- $\beta$-caroteno, 9-cis- $\beta$-caroteno e 13-cis- $\beta$ caroteno) (300-550 nm), que quando dispersos em etanol apresentam dois picos entre 240 a $280 \mathrm{~nm}$ e entre 300 a $550 \mathrm{~nm}$ (Bernardi; Nicolini; Nicolini, 2017).

Uma banda em 661,4 nm larga e fraca (Fig. 2) é observada sugerindo a presença de carotenóides acíclicos que possuem absorção máxima em comprimentos de ondas maiores que os demais carotenóides cíclicos com mesmo número de duplas ligações conjugadas (DLC), onde quando a conjugação se estende ao anel, ocorrem tensões de anel, pois as DLC dos anéis não são coplanares com o resto da cadeia, ou a presença de pigmentos clorofilianos $a$ e $b(600$ a $700 \mathrm{~nm})$ (Zeraik e Yariwake, 2008; Maestrin et al., 2009; Silva et al., 2014). Isso pode ser constatado em ambas as (Fig. 2 e 3) entre a varredura de 900 a $400 \mathrm{n}$ e o comprimento de ondas crítico entre 220 a $400 \mathrm{~nm}$, onde o mesmo é discutido por Violante et al. (2009) avaliando diferentes extrato vegetais com potencial agente fotoprotetor.

$\mathrm{O}$ extrato de uvaia demonstrou absorver a energia ultravioleta UVC, que compreende entre 100 a
$290 \mathrm{~nm}$. Vários extratos apresentam tendência de absorção nos comprimentos de ondas entre 290 a $315 \mathrm{~nm}$ (UVB) e entre 315 a $400 \mathrm{~nm}$ que compreende absorção em UVA (Schauder; Ippen, 1997; Aikens, 2006; Violante et al., 2008). Conforme discutido por Souza et al. (2005), as radiações ultravioletas (UV) são fontes extremamente energéticas, sendo divididas em UVA, UVB e UVC.

De acordo com Souza et al. (2013), Carvalho et al. (2015), e Vieira e Orlanda (2018), as inúmeras classes fitoquímicas como os flavonoides, taninos, alcaloides e vitaminas $\mathrm{C}$ e $\mathrm{E}$ apresentam alta efetividade como agentes fotoprotetores cutâneos com maior amplitude em soluções tópicas com fator de proteção solar (FPS).

As plantas representam um importante banco de moléculas fitoquímicas de grande importância para o homem, a fotoproteção apresenta vários estudos com extratos vegetais, visto que, devido a sua composição fitoquímica formam uma camada protetora sobre a derme prevenindo as queimaduras causadas pelas fontes de radiação ultravioleta. As fontes UV, são deletérias para as células, causando a nível celular o desenvolvimento de cânceres como melanoma e o envelhecimento precose (Orlanda; Vale, 2015; Vieira e Orlanda, 2018).

$\mathrm{Na}$ Figura 4, está apresentada a porcentagem de hemólise sobre eritrócitos humanos 5\% a partir de concentrações decrescentes de extrato hidroetanólico do fruto de E. pyriformis

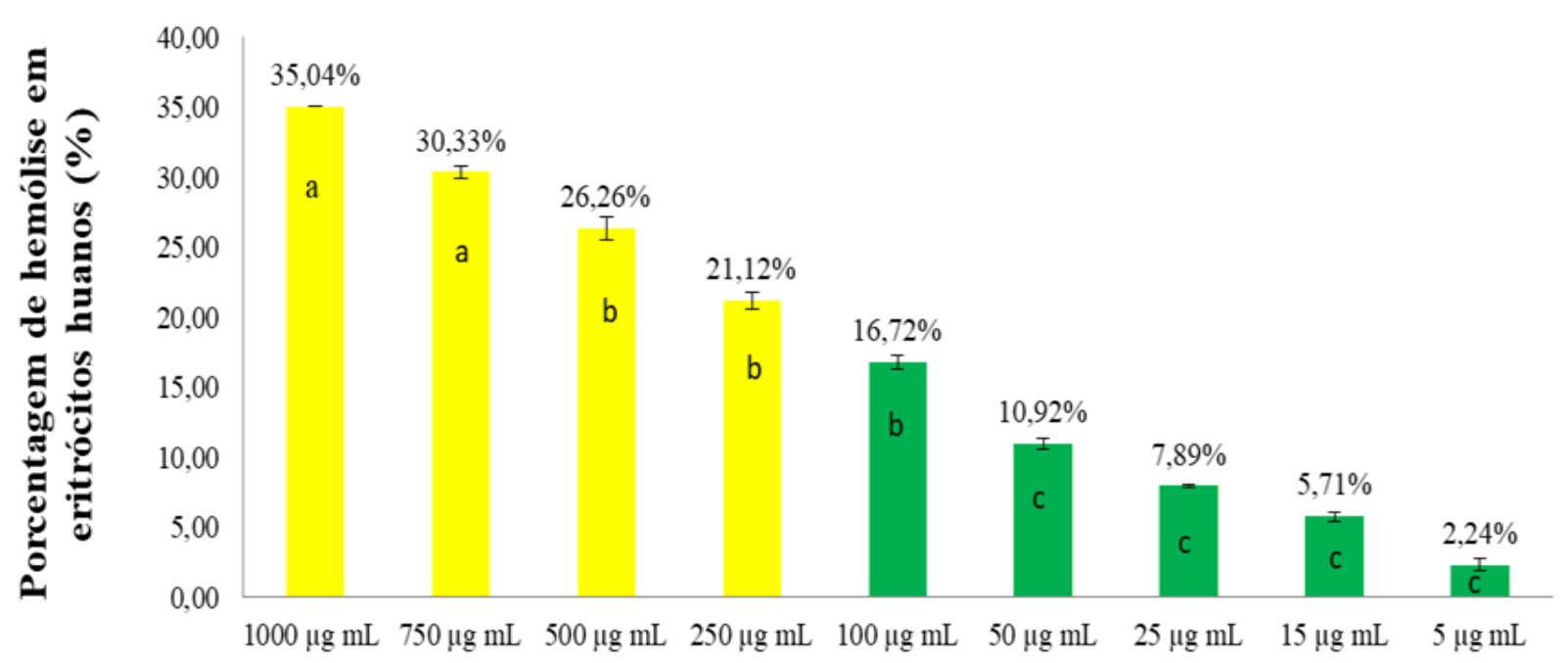

Concentração do extrato de $E$. pyriformis

Figura 4 - Ensaio de atividade hemolítica em eritrócitos humanos sobre o extrato hidroetanólico de Eugenia pyriformis. 
Conforme se observa na figura 4 , a atividade hemolítica em eritrócitos humanos foi constatada sobre o extrato dos frutos de uvaia, associando este efeito com a presença de saponinas hemolíticas, onde demonstrou, no entanto, ser baixo com hemólise de $35 \%$ na maior concentração de 1000 $\mu \mathrm{g} \mathrm{mL}^{-1}$ e de $2 \%$ na menor concentração de $5 \mu \mathrm{g}$ $\mathrm{mL}^{-1}$.

De acordo com Nofiani et al. (2011), e Ramos et al. (2020), processos hemolíticos acima de $40 \%$ são considerados altos. O baixo potencial de citotoxicidade sobre eritrócitos humanos garante o uso do extrato hidroetanólico dos frutos de uvaia para diversos fins, como por exemplo, solução fotoprotetora na linha natural.

\section{CONCLUSÕES}

O extrato da casca com polpa do fruto de Eugenia pyriformis apresentaram resultados importantes que irão constituir no conhecimento sobre o extrato hidroetanólico $70 \%$ do fruto tanto para os parâmetros físico-químicos, quanto para as análises de conteúdos de flavonoides, taninos e açúcares naturais, bem como, nas atividades de fotoproteção e de citotoxicidade sobre hemólise de eritrócitos humanos, onde apresentaram resultados satisfatórios para o uso do extrato em linhas farmacêuticas de uso tópico como fotoprotetores solares.

Novos estudos deverão ser realizados avaliando outras atividades biológicas que enriquecerão o conhecimento sobre essa espécie frutífera pertencente ao gênero Eugenia e família Myrtaceae.

\section{AGRADECIMENTOS}

Ao Instituto Federal Goiano; a Universidade Estadual de Goiás, Campus Santa Helena de Goiás; aos laboratórios de Química Geral e Inorgânica, Química Orgânica, Química Tecnológica; aos órgãos de fomento em pesquisa CAPES, CNPq e FINEP.

\section{REFERÊNCIAS BIBLIOGRÁFICAS}

Aikens P. Nanomateriais proporcionam proteção solar de amplo espectro. Cosmetics \& Toiletries, v. 18, p. 60-64, 2006.

Alves, M. M.; Pereira, M. A. S.; Pereira, O. S.; França, S. C.; Bertoni, B. W. Caracterização química de tinturas e extratos secos de plantas medicinais do Cerrado por cromatografia em camada delgada. Scientia Plena, v. 7, n. 12, 2011.

https://scientiaplena.emnuvens.com.br/sp/article/view/159

Amorim SL, da Silva LB, Barata JS, Pereira MAFM, de Oliveira ACP, Athayde ACR. Prospecção fitoquímica da
Carapa guianensis (Meliaceae) e Uncaria guianensis (Rubiaceae) com vista á atividade anti-helmíntica sobre nematódeos gastrintestinais de pequenos ruminantes. Scientia Naturalis, v.2, n.1, p.133-142, 2020.

https://periodicos.ufac.br/index.php/SciNat/article/view/35 85

Arantes AA, Monteiro R. A família Myrtaceae na Estação Ecológica do Panga, Uberlândia, Minas Gerais, Brasil. Lundiana, v.3, n.2, p.111-127, 2002.

https://periodicos.ufmg.br/index.php/lundiana/article/view/ 21804

Bernardi F, Nicolini KP, Nicolini J. Estudo fitoquímico de Hydrangea sp. por meio de métodos clássicos de análise por espectroscopia no ultravioleta visível (UV-Vis) e cromatografia em coluna e em papel. Infarma, v.29, n.1, p.6880, 2017. https://core.ac.uk/download/pdf/205665347.pdf

Bianchini CB, Arriola NDA, Seraglio SKT, Costa ACO, Ribeiro DHB, Komatsu RA, Machado BD, Amboni RDMC, Fritzen-Freire CB. Influence of pasteurization on the chemical, physical, and microbiological characteristics of uvaia pulp (Eugenia pyriformis Cambess). Research, Society and Development, v.9, n.7, p. e993975192, 2020. https://www.rsdjournal.org/index.php/rsd/article/view/519 2

Cala-Calviño L, Cala DJ, Fernández RG, Barrientos AB, Sánchez-Hachavarría ME, Vadell HC. Estudio farmacognóstico preliminar de la especie Annona squamosa L. Revista Cubana de Plantas Medicinales, v.23, n.2, 2018. http://www.revplantasmedicinales.sld.cu/index.php/pla/arti cle/view/637

Carvalho JC, Garcia PSP, Vignol SR, Pedriali CA. Estudo do impacto da utilização de ativos vegetais em fotoprotetores. InternacEHS - Saúde, Meio Ambiente e Sustentabilidade, v. 10, n. 2 , p. $62-82,2015$. https://www.sp.senac.br/blogs/InterfacEHS/wpcontent/uploads/2015/12/150_artigo_alteracoes.pdf

Catelan TBS, Gaiola L, Duarte BF, Cardoso CAL. Evaluation of the in vitro photoprotective potential of ethanolic extracts of four species of the genus Campomanesia. Journal of Photochemistry and Photobiology B: Biology, v.197, 2019.

https://doi.org/10.1016/j.jphotobiol.2019.04.009

Dacie JV, Lewis SM, Catovsky D. Blood cell cytochemistry and supplementary techniques. Practical Hematology, Churchill Livingstone, Edinburgh, p.120-148, 1975.

Dexheimer GM, Pozzobon A. Atividade biológica de plantas da família Myrtaceae: revisão sistemática de artigos entre 1989 e 2015. Revista Cubana de Plantas Medicinales, v.22, n.2, 2017.

http://www.revplantasmedicinales.sld.cu/index.php/pla/arti cle/view/534

Domínguez OGP, Pesantes KEB, Martínez MM, Gaitén YG. Estudio químico y evaluación biológica del extracto etanólico de Allium schoenoprasum L. Regel \& Tiling (Cebollín). Revista Cubana de Farmacia, v.52, p.1, p. e98, 2019. http://revfarmacia.sld.cu/index.php/far/article/view/98

Ferreira PM, Farias DF, Viana MP, Souza TM, Vasconcelos IM, Soares BM. Study of the antiproliferative potential of seed extracts from Northeastern Brazilian plants. Anais da Academia Brasileira de Ciências, v.83, n.3, p.1045-1058, 2011. https://doi.org/10.1590/S0001-37652011005000017 
Franzen FL, Fries LLM, de Oliveira MSR, Lidório HF, Menegaes JF, Lopes SJ. Teor e rendimento de extratos de flores obtidos por diferentes métodos e períodos de extração. Acta Iguazu, v.7, n.1, p.9-21, 2018. https://doi.org/10.48075/actaiguaz.v7i1.16765

Kauffmann C, Soares APV, Arossi K, Pacheco LA, Buhl B, Freitas EM, Hoehne L, Castro LC, Gnoatto SCB, Ethur EM. Potencial antimicrobiano e antibiofilme in vitro de espécies do gênero Eugenia, Myrtaceae, nativas do Sul do Brasil. Caderno Pedagógico, v.14, n.2, p.110-127, 2017. http://dx.doi.org/10.22410/issn.19830882.v14i2a2017.1512

Lazzarotto-Figueiró J, Capelozzo AP, Schindler MSZ, Fossá JFC, Albeny-Simões D, Zanatta L, Oliveira JV, Dal Magro J. Antioxidant activity, antibacterial and inhibitory effect of intestinal disaccharidases of extracts obtained from $\mathrm{Eu}$ genia uniflora L. seeds. Brazilian Journal of Biology, v.81, n.2, p.291-300, 2021. https://doi.org/10.1590/15196984.224852

Lucas EJ, Harris SA, Mazine FF, Belsham SR, Nic Lughadha EM, Telford A, Gasson PE, Chase MW. Suprageneric phylogenetics of Myrteae, the generically richest tribe in Myrtaceae (Myrtales). Taxon, v.56, p.1105-1128, 2007. https://doi.org/10.2307/25065906

Lucas EJ, Matsumoto K, Harris SA, Nic lughadha EM, Benardini B, Chase MW. Phylogenetics, Morphology, and Evolution of the Large Genus Myrcia s. 1. (Myrtaceace). International Journal of Plant Sciences, v.172, p.915-934, 2011. https://doi.org/10.1086/660913

Machado CC, Silva PA, Souza JF, Souza ACF. Efeito do extrato aquoso de alho Allium sativum L. sobre a atividade antibacteriana de antibióticos utilizados contra Staphylococcus aureus. Arquivos Científicos, v. 2, n. 2, p. 111-118, 2019. https://doi.org/10.5935/2595-

4407/rac.immes.v2n2p111-118

Maestrin APJ, Neri CR, Oliveira KT, Serra AO, Iamamoto, Y. Extração e purificação de clorofila $A$, da alga Spirulina maxima: um experimento para os cursos de química. Química Nova, v.32, n.6, p.1670-1672, 2009. https://doi.org/10.1590/S0100-40422009000600054

Menezes Filho ACP, Mariana CS, Castro CFS. Estudo fitoquímico, bioativo, fotoprotetor e físico-químico do extrato floral de algodãozinho do Cerrado [Cochlospermum regium Schrank. (Pilg.)] - Bixaceae. Arquivos Científicos, v. 3, n. 2, 2020.

http://arqcientificosimmes.emnuvens.com.br/abi/article/vie w/375

Morrison M, Asiedu EA, Stuchbury T, Powell AA. Determination of lignin and tannin contents of cowpea seed coats. Annals of Botany, v. 76, n. 1, p. 287-290, 1995. https://doi.org/10.1006/anbo.1995.1097

Nofiani R, Kurniadi R, Ardiningsih P. Antimicrobial, antioxidant, hemolytic activities and toxicity of ethyl acetate extract from an unidentified coral-associated Fungus, Aspergillus brevipes RK06. Indonesian Journal of Cancer Chemoprevention, v.2, n.2, p.212-216, 2011. http://dx.doi.org/10.14499/indonesianjcanchemoprev2iss2p p212-216

Orlanda JFF. Vale VV. Análise fitoquímica e atividade fotoprotetora de extrato de Euphorbia tirucalli Linneau (Euphorbiaceae). Revista Brasileira de Plantas Medicinais, v. 17, n. 4, supl. I, p. 730-736, 2015.

http://dx.doi.org/10.1590/1983-084X/14_012

Paunović MG, Ognjanović BI, Matić MM, Štajn AŠ, Saičić ZS. Protective effects of quercetin and vitamin $\mathrm{C}$ against nicotine-induced toxicity in the blood of Wistar rats. Archives of Industrial Hygiene and Toxicology, v. 67, n. 4, p. 304-310, 2016. https://doi.org/10.1515/aiht-2016-67-2795

Plucenio LP, Silva VL. Propriedades bioativas e antioxidantes de uvaias (Eugenia pyriformis Cambess) cultivadas na região de Pelotas, Rio Grande do Sul. In: Salão UFRGS, 2017. SIC - XXIX Salão de Iniciação Científica da UFRGS, Campus do Vale, Brasil, 2017.

Ramos RM, Feitosa JM, Lima VS, Santos RC, de Oliveira AM, Soares LAL, da Silva GC. Estudo comparativo da composição fitoquímica, citotoxicidade e potencias antioxidante e fotoprotetora da casca e folha de Erythrina velutina. Brazilian Journal of Development, v.6, n.6, p.3314033158, 2020. https://doi.org/10.34117/bjdv6n6-026

Schauder S, Ippen H. Contact and photocontact sensitivity to sunscreen. Contact Dermatitis, v.37, p.221-232, 1997. https://doi.org/10.1111/j.1600-0536.1997.tb02439.x

Sganzerla WG, Beling PC, Ferreira ALA, Azevedo MS, Ferrareze JP, Komatsu RA, Nunes MR, Veeck APL. Geographical Discrimination of uvaia (Eugenia pyriformis Cambess) by principal component analysis. Journal of the Science of Food and Agriculture, v.99, n.15, p.6778-6787, 2019. https://doi.org/10.1002/jsfa.9961

Silva AT, Mazine FF. A família Myrtaceae na floresta nacional de Ipanema, Iperó, São Paulo, Brasil. Rodriguésia, v.67, n.1, p.203-223, 2016. http://dx.doi.org/10.1590/21757860201667110

Silva DA, Costa RG, Borges LL, Alves SF. Preparação e caracterização físico-química do extrato hidroetanólico de Genipa americana Linnaeus. Revista Eletrônica de Farmácia, v.16, n. E, p.1-7, 2019.

Silva RN, Monteiro VN, Alcanfor J.D'AX, Assis EM, Asquieri ER. Comparação de métodos para a determinação de açúcares redutores e totais em mel. Ciência e Tecnologia de Alimentos, v.23, n.3, p.337-341, 2003. http://dx.doi.org/10.1590/S0101-20612003000300007

Silva ST, Rodrigues MA, Pacheco FV, Alvarenga ICA, Bertolucci SKV, Pinto JEBP. Crescimento e pigmentos fotossintéticos de pimenta-de-macaco in vitro, sob diferentes luminosidades. Horticultura Brasileira, v.31, p.S2683S2689, 2014. http://www.abhorticultura.com.br/EventosX/Trabalhos/EV _7/A5815_T9252_Comp.pdf

Souza FP, Campos GR, Packer JF. Determinação da atividade fotoprotetora e antioxidante em emulsões contendo extrato de Malpighia glabra L. - acerola. Revista de Ciências Farmacêuticas Básica e Aplicada, v.34, n.1, p.69-77, 2013. http://rcfba.fcfar.unesp.br/index.php/ojs/article/view/239

Souza TM, Santos LE, Moreira RRD, Rangel VLBI. Avaliação da atividade fotoprotetora de Achillea millefolium $\mathrm{L}$. (Asteraceae). Revista Brasileira de Farmacognosia, v.15, n.1, p.36-38, 2005. https://doi.org/10.1590/S0102$695 \times 2005000100008$

Stieven AC, Moreira JJS, Silva CF. Óleos essenciais de uvaia (Eugenia pyriformis Cambess): avaliação das atividades 
microbiana e antioxidante. Eclética Química, v.34, n.3, p.7-13, 2009. https://doi.org/10.1590/S010046702009000300001

Takao LK, Imatomi M, Gualtieri SCJ. Antioxidant activity and phenolic content of leaf infusions of Myrtaceae species from Cerrado (Brazilian Savanna). Brazilian Journal of Biology, v.75, n.4, p.948-952, 2015.

https://doi.org/10.1590/1519-6984.03314

Vieira CBS, Orlanda JFF. Atividade antioxidante e fotoprotetora do extrato etanólico de Ocimum gratissimum L. (alfavaca, Lamiaceae). Revista Cubana de Plantas Medicinales, v. 23, n.3, 2018.

http://revplantasmedicinales.sld.cu/index.php/pla/article/vi ew/626

Violante IMP, Souza IM, Venturini CL, Ramalho AFS, Santos RAN, Ferrari M. Avaliação in vitro da atividade fotoprotetora de extratos vegetais do Cerrado de Mato Grosso. Revista Brasileira de Farmacognosia, v.19, n.2A, p.452-457, 2009. https://doi.org/10.1590/S0102$695 \times 2009000300020$

Wagner H, Blandt S. Plant drug analysis: a thin layer chromatography. Atlas 2 Ed., Germany: Springer, 2001.

Zapata HB, Palacios GP, Moreno LPP, Llanos CAH. Estudio etnobotánico de plantas medicinales en tres municipios de Antioquia, Colombia. Revista Cubana de Plantas Medicinales, v.23, n.4, 2018.

http://www.revplantasmedicinales.sld.cu/index.php/pla/arti cle/view/665

Zeraik ML, Yariwake JH. Extração de $\beta$-caroteno de cenouras: uma proposta para disciplinas experimentais de química. Química Nova, v.31, n.5, p.1259-1262, 2008. http://dx.doi.org/10.1590/S0100-40422008000500058

Zhishen J, Mengcheng T, Jianming W. The determination of flavonoid contents in mulberry and their scavenging effects on superoxide radicals. Food Chemistry, v.64, p.555-559, 1999. https://doi.org/10.1016/S0308-8146(98)00102-2 